\title{
The Visual Word Form Area natively processes shape sequences: Implications for developmental dyslexia
}

*Carol Whitney ${ }^{\mathrm{a}}$,

Paddy Ross ${ }^{\mathrm{b}}$,

Zhiheng Zhou',

Lars Strother ${ }^{\mathrm{c}}$

annaffiliated Researcher, Silver Spring, MD, USA

10

bepartment of Psychology, Durham University, Durham, UK

'Department of Psychology, University of Nevada, Reno, NV, USA

15 *Correspondence:

Dr. Carol Whitney

cwhitney10@gmail.com 


\section{Abstract}

The Visual Word Form Area (VWFA) is a cortical region that adapts to support fluent word recognition. Surprisingly, the region of ventrolateral occipitotemporal cortex that becomes VWFA is specialized for

25 processing the motion of inanimate objects that change shape. Such motion is neurally analyzed as a temporal sequence of shape 'snapshots'. We have proposed that the VWFA develops in this region because letter representations are serially activated in occipitotemporal cortex during typical reading acquisition. Therefore, the region that analyzes inanimate shape sequences is recruited to recognize letter sequences. We discuss the implications of this account for developmental dyslexia. In particular,

30 inability to focus attention down to a single letter may preclude the serial letter selection that typically drives VWFA formation. Such a deficit would also interfere with acquisition of cortical letter-phoneme connections. Instead, compensated dyslexics employ the ventromedial object-recognition system for whole-word recognition, and the subcortical procedural system for phonological decoding. 


\section{Background}

35 The Visual Word Form Area (VWFA) in occipitotemporal cortex (OTC) supports fluent word recognition (Dehaene \& Cohen, 2011), and lack of OTC specialization for reading is commonly observed in developmental dyslexia (Danelli et al., 2017; Monzalvo, Fluss, Billard, Dehaene, \& Dehaene-Lambertz, 2012; Paulesu, Danelli, \& Berlingeri, 2014; Richlan, Kronbichler, \& Wimmer, 2009). In skilled readers, the VWFA is positioned at a similar location across subjects (Vinckier et al.,

40 2007). Understanding how and why the VWFA normally develops where it does is necessary for understanding its impaired formation in dyslexia.

Many researchers assume that VWFA location is driven by pre-existing connectivity to language areas (Dehaene \& Dehaene-Lambertz, 2016; Hannagan, Amedi, Cohen, Dehaene-Lambertz, \& Dehaene, 2015; Pugh et al., 2013; Saygin et al., 2016; Schlaggar \& McCandliss, 2007; Stevens, Kravitz, Peng,

45 Tessler, \& Martin, 2017; Yeatman, Rauschecker, \& Wandell, 2013). However, the cortical region typically identified as the VWFA consists of two areas with different response profiles, namely the VWFA proper on the Occipitotemporal Sulcus/lateral Fusiform Gyrus, and the Lateral Inferotemporal Multimodal Area (LIMA) on the Inferior Temporal Gyrus. (Henceforth, VWFA will refer to the VWFA proper.) Both areas respond to visual words, but only the LIMA responds for tasks on auditory words

50 (Cohen, Jobert, Le Bihan, \& Dehaene, 2004; Danelli et al., 2017, 2013). The LIMA is thought to provide an interface between the visual and language systems (Cohen et al., 2004; Price \& Devlin, 2011). Therefore, LIMA location is indeed likely determined by connectivity to the language system. What then drives VWFA location within visual cortex?

A priori, one would expect visual word recognition to be carried out via the object-recognition system.

55 Surprisingly, the VWFA is not located in the cortical region specialized for recognition of inanimate static objects; rather, the VWFA forms in an area that processes the motion of objects undergoing changes in shape (dynamic motion) (Whitney, Ross, Zhou, \& Strother, 2019). Why would this be? Dynamic object motion is analyzed as a temporal sequence of shapes, and letter processing is obviously serial in early reading acquisition. Therefore, the shape-sequence area is recruited to encode and 60 recognize letter sequences (Whitney et al., 2019).

The present article discusses the implications of this analysis for the etiology of developmental dyslexia. We propose that inability to narrow visual attention to individual letters precludes the sequential activation of occipitotemporal letter representations which normally drives VWFA 
development. Before presenting this account of dyslexia, we review Whitney et al. (2019) in more

65 detail, and summarize ongoing experimental research.

\section{Occipitotemporal Organization}

OTC is organized around the properties of motion, shape, and animacy. Figure 1 illustrates this organization, and defines abbreviations used in the following text. The region outlined in gold is the Lateral Occipital Complex, which responds more strongly to images of intact than scrambled objects

70 (Grill-Spector, 2003; Hasson, Harel, Levy, \& Malach, 2003; Jastorff \& Orban, 2009; Silson et al., 2013), indicating specialization for shape processing. The green- and blue-filled oval regions respond more strongly to moving than still objects/humans, indicating specialization for action perception and guidance (Beauchamp, Lee, Haxby, \& Martin, 2002, 2003; Costantini et al., 2005; Jastorff \& Orban, 2009).

75 Selectivity for motion and shape yields three OTC zones. The dorsolateral zone (dlOTC), which is sensitive only to motion, lies on the STS and MTG. The motion- and shape-sensitive ventrolateral zone (vlOTC) extends from the ITS to the lateral FG. The ventromedial zone (vmOTC), which is sensitive only to shape, lies on the medial FG and CoS.

The medial FG responds more strongly to inanimate objects than humans/animals (Beauchamp et al.,

80 2002; Konkle \& Caramazza, 2013; Stevens, Tessler, Peng, \& Martin, 2015; Wiggett, Pritchard, \& Downing, 2009). Object shape and color are integrated in the mid medial FG (Lafer-Sousa, Conway, \& Kanwisher, 2016). Hence, the vmOTC supports visual object recognition.

The motion-sensitive lateral OTC is comprised of areas with various preferences for animacy and type of motion. Animate entities move with changing shape (dynamic motion, such as a human walking),

85 while inanimate objects often move with a fixed shape (rigid motion, such as a hammer in use). The green-filled areas respond more strongly to rigid motion than to still objects. The posterior green-filled area is $\mathrm{hMT}+$, the human retinotopic motion complex. The anterior green-filled area, which we have dubbed the Lateral Object Area (LOA), responds most strongly to inanimate objects undergoing rigid motion (Beauchamp et al., 2002, 2003). For static images, the LOA responds preferentially to tools and small objects (Bracci, Cavina-Pratesi, Connolly, \& Ietswaart, 2016; Bracci, Cavina-Pratesi, Ietswaart, Caramazza, \& Peelen, 2012; Hasson et al., 2003; Ishai, Ungerleider, Martin, Schouten, \& Haxby, 1999). The left LOA responds more strongly to images of hands than other body parts (Bracci et al., 2012). These properties suggest that the left LOA is specialized for encoding interactions with objects. 

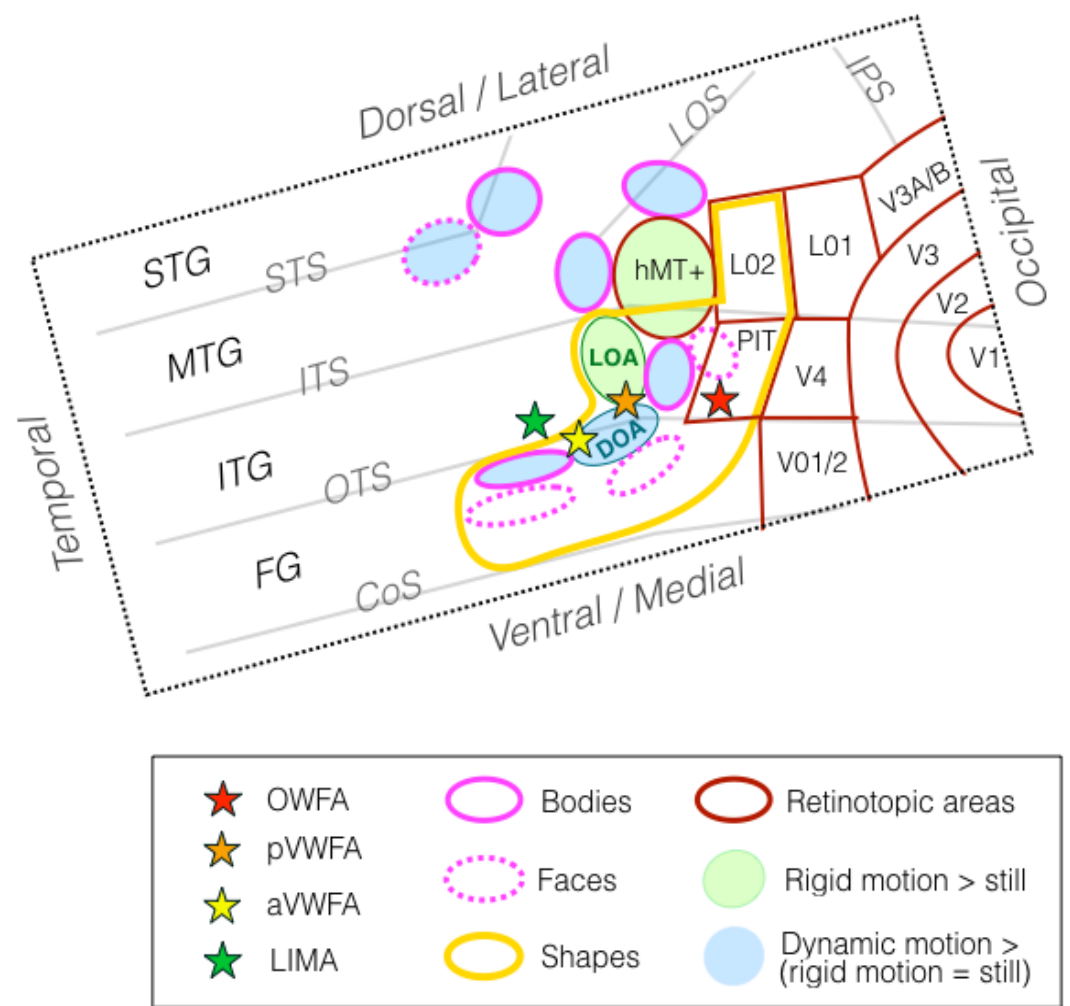

Figure 1: Idealized flattened occipitotemporal cortex showing approximate locations of regions selective for motion, shapes, and animacy. Locations of retinotopic areas relative to gyri are taken from Abdollahi et al. (2014). Locations of categorical regions based on references cited in the text. Abbreviations: STG Superior Temporal Gyrus; STS Superior Temporal Sulcus; MTG Middle Temporal Gyrus; ITS Inferior Temporal Sulcus; ITG Inferior Temporal Gyrus; FG Fusiform Gyrus; CoS Collateral Sulcus; PIT phPIT, putative human homolog of macaque Posterior Inferotemporal area; OWFA Occipital Word Form Area; $p$ VWFA posterior VWFA; aVWFA anterior VWFA; LIMA Lateral Inferotemporal Multimodal Area; LOA Lateral Object Area (defined in text); DOA Dynamic Object Area (defined in text).

The blue-filled areas respond to dynamic motion (vs still objects or rigid motion), but not to rigid motion (Beauchamp et al., 2002, 2003; Beauchamp \& Martin, 2007; Jastorff \& Orban, 2009).

Dynamic motion can be neurally analyzed in two ways. One is that motions of small edge segments are evaluated, and these local motion vectors are integrated. The other is that whole-object shape is

100 evaluated at successive time points, and these 'snapshots' are integrated. For example, a point-light stimulus would preferentially drive motion-vector analysis, while a slow image sequence (say at 4 frames/sec) would drive shape-based analysis. In line with their selectivities, dlOTC employs motion- 
vector analysis, and vlOTC shape-based analysis (Beauchamp et al., 2003; Jastorff \& Orban, 2009; Vangeneugden, Peelen, Tadin, \& Battelli, 2014).

105 The blue-filled areas with solid magenta outlines respond more strongly to animals and human bodies than inanimate objects (Konkle \& Caramazza, 2013; Wiggett et al., 2009). The three such regions adjacent to hMT+ comprise the Extrastriate Body Area (EBA) (Downing, Jiang, Shuman, \& Kanwisher, 2001; Weiner \& Grill-Spector, 2013). The FG region is the Fusiform Body Area (FBA) (Peelen \& Downing, 2005). Areas that respond preferentially to faces are located near body areas

110 (Kanwisher, McDermott, \& Chun, 1997; Liu, Harris, \& Kanwisher, 2010; Polosecki et al., 2013). Another blue-filled region falls between the FBA and ventral EBA. Unlike the adjacent Body Areas, this region is not selective for human dynamic motion (Beauchamp \& Martin, 2007; Jastorff \& Orban, 2009). Unlike the nearby LOA, this region is not sensitive to rigid motion (Beauchamp \& Martin, 2007; Spiridon, Fischl, \& Kanwisher, 2006). So what is the selectivity of this area? Bilaterally, this

115 region responds more robustly to dynamic motion of invented 'Creatures' than humans, and is the only such region to do so (Pyles, Garcia, Hoffman, \& Grossman, 2007). It responds more strongly to dynamic motion of hands or scissors than static hands or scissors (Costantini et al., 2005). In the left hemisphere only, this region responds more strongly to dynamic motion of actual objects than humans (Whitney et al., 2019).

120 Given this region's response profile and location between areas sensitive to human whole-body motion, we suggest that it is specialized for the analysis of dynamic motion of other stimuli, such as animals, forearms, and inanimate objects. The right hemisphere may be specialized for animals, whereas the left hemisphere may be specialized for forearms and objects, like the left LOA. We have dubbed this lefthemisphere region the Dynamic Object Area (DOA). The location of the DOA within vlOTC indicates

125 that dynamic object motion is analyzed as a temporal sequence of shapes.

\section{Orthographic Processing}

In Fig. 1, stars illustrate the centers of areas involved in orthographic analysis. The posterior VWFA houses sublexical orthographic representations (Dehaene et al., 2004; Lerma-Usabiaga, Carreiras, \& Paz-Alonso, 2018; Thesen et al., 2012), while the anterior VWFA encodes whole words (Glezer, Jiang,

130 \& Riesenhuber, 2009; Glezer, Kim, Rule, Jiang, \& Riesenhuber, 2015). The posterior VWFA is sensitive to rigid motion (random movement of a Gabor patch), while the anterior VWFA is not (Danelli et al., 2017, 2013). We use pVWFA to denote the sublexical part of the VWFA; some, but not 
necessarily all, of the pVWFA is sensitive to rigid motion. aVWFA will denote the lexical portion of the VWFA, which is insensitive to rigid motion.

135 A more posterior region, the Occipital Word Form Area (OWFA), is also specialized for visual word recognition, but is not motion-sensitive (Strother, Coros, \& Vilis, 2016; Strother, Zhou, Coros, \& Vilis, 2017). The OWFA is located between V4 and hMT+, likely corresponding to a shape-selective retinotopic region (phPIT) that is the putative human analog of the macaque Posterior Inferotemporal area (Kolster, Peeters, \& Orban, 2010).

140 A priori, one would expect visual words to be identified in cortex specialized for recognition of static inanimate objects, namely vmOTC. Indeed, recognition of individual letters activates vmOTC (James, James, Jobard, Wong, \& Gauthier, 2005). However, the aVWFA is not located in vmOTC, but rather falls in the OTS in vlOTC (Cohen et al., 2004; Danelli et al., 2017, 2013), in the region of the DOA. Why would visual words be recognized in cortex specialized for encoding dynamic object motion? In

145 the early phases of reading acquisition, letters are overtly serially processed. Hence, the VWFA develops in cortex specialized for encoding temporal sequences of inanimate shapes.

Orthographic processing during reading acquisition can be divided into three phases, based on saccade distance and magnitude of the length effect in naming short words (2-5 letters). When children begin to read (first phase), saccade distance is one letter and the length effect is on the order of $200 \mathrm{~ms} / \mathrm{letter}$,

150 indicating that children fixate each letter of a word (De Luca, Zeri, Spinelli, \& Zoccolotti, 2010; Zoccolotti et al., 2005). After about six months of reading instruction (second phase), the length effect drops dramatically, to $\sim 50 \mathrm{~ms} /$ letter, and saccade distance increases up to about five letters (De Luca et al., 2010; Marinus \& de Jong, 2010; Zoccolotti et al., 2005), suggesting that children covertly shift attention across letters within a fixation. Note that letter processing is obviously serial during the first

155 and second phases. After three or four years of reading experience (third phase), the length effect becomes negligible (De Luca et al., 2010; Gagl, Hawelka, \& Wimmer, 2015; Marinus \& de Jong, 2010; Zoccolotti et al., 2005), indicating skilled orthographic processing.

In the following, capitalized words will indicate neural representations; e.g., Letter denotes a neural encoding of a letter. We propose that Letters develop in the LOA. Correspondingly, the initial ventral 160 OTC specialization for reading forms in a lateral tool-selective area (Dehaene-Lambertz, Monzalvo, \& Dehaene, 2018). We assume that a letter is selected by zooming attention to that letter; as a result, only one Letter is highly active at a time. We propose that the DOA is recruited to recognize the Letter 
sequences generated by serial letter selection, forming the lexical pathway. In particular, Open-Bigrams in the posterior DOA (pDOA) encode temporally-ordered pairs of Letters, and Visual Word Forms in 165 the anterior DOA (aDOA) recognize a word as a set of Open-Bigrams (Whitney, 2001; Whitney \& Marton, 2013). Thus, the pVWFA develops in the LOA and pDOA, and the aVWFA in the aDOA. The overlap of the pVWFA and LOA accounts for the observed rigid-motion sensitivity (Danelli et al., 2013). Letters also develop connections to Phonemes in the STS, forming the sublexical phonological pathway (Blomert, 2011; Dehaene-Lambertz et al., 2018).

170 Note that serial Letter activation provides an abstract (non-retinotopic) encoding of letter order. The lexical and sublexical pathways develop under this encoding, and likely come to require it. Therefore, this encoding should be preserved in the third orthographic phase. However, the absence of a length effect has led most researchers to erroneously conclude that letters are processed fully in parallel on the lexical pathway; Whitney (2018) explains how serial Letter activation can fail to generate a length

175 effect. We propose that the top-down attention shifts of the second phase drive perceptual learning in high-level retinotopic cortex, yielding bottom-up sequential activation across a retinotopic representation of case-specific letter identities (Retinotopic-Letters), at the rate of $\sim 16 \mathrm{~ms} / \mathrm{letter}$ (Whitney \& Marton, 2013). Hence, the OWFA develops in phPIT. The Retinotopic-Letters serially activate the non-retinotopic, case-invariant Letters in turn. Figure 2 summarizes the orthographic 180 phases. The top panel of Figure 3 illustrates the sublexical and lexical pathways in skilled readers. 


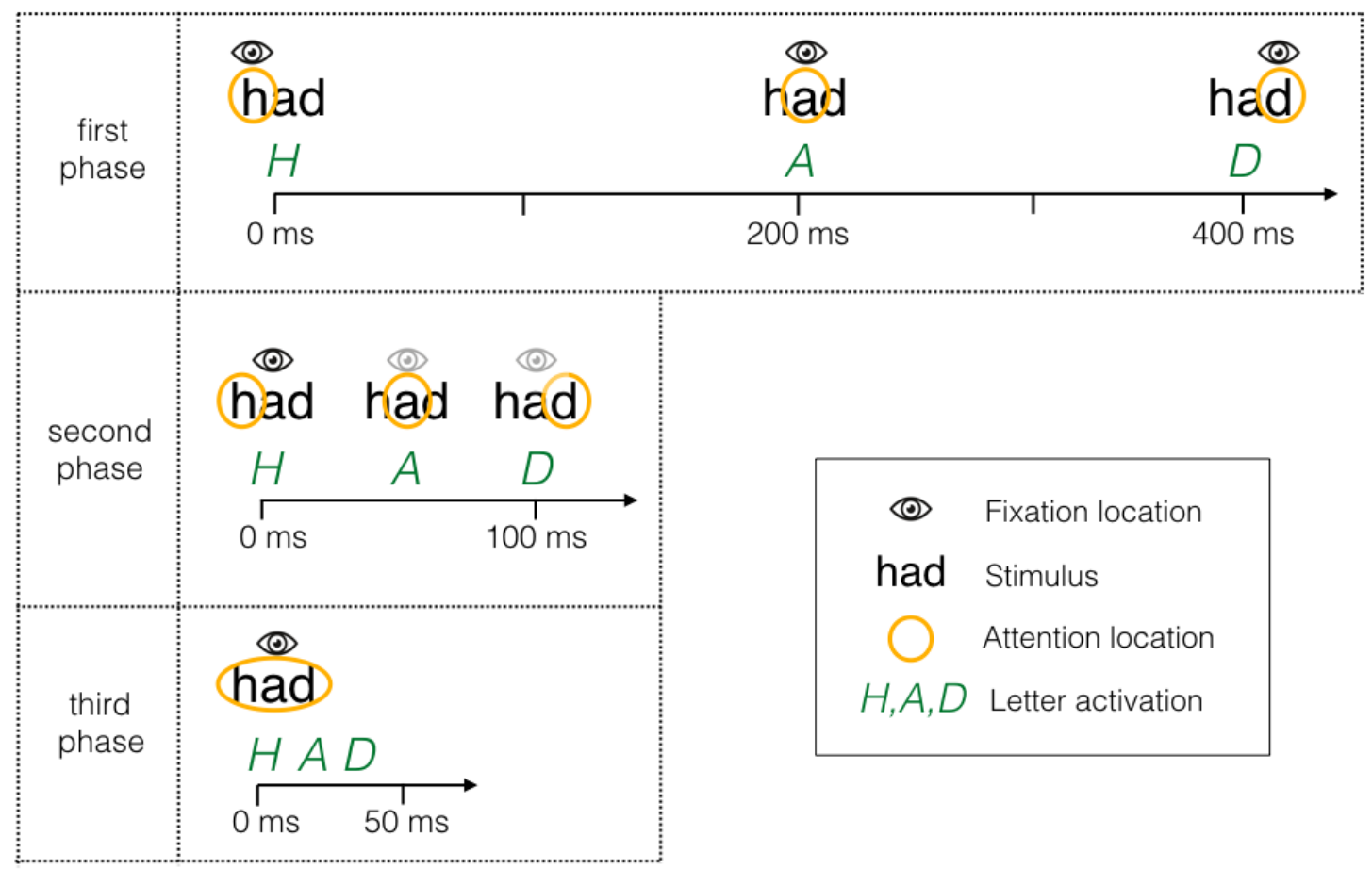

Figure 2: The proposed orthographic phases in typical readers. First phase: serial letter processing via saccades. Second phase: letters selected via top-down shifts of covert attention. Third phase: whole word is attended, and acquired bottom-up processing yields serial Letter activation. 


\section{Skilled Reader}

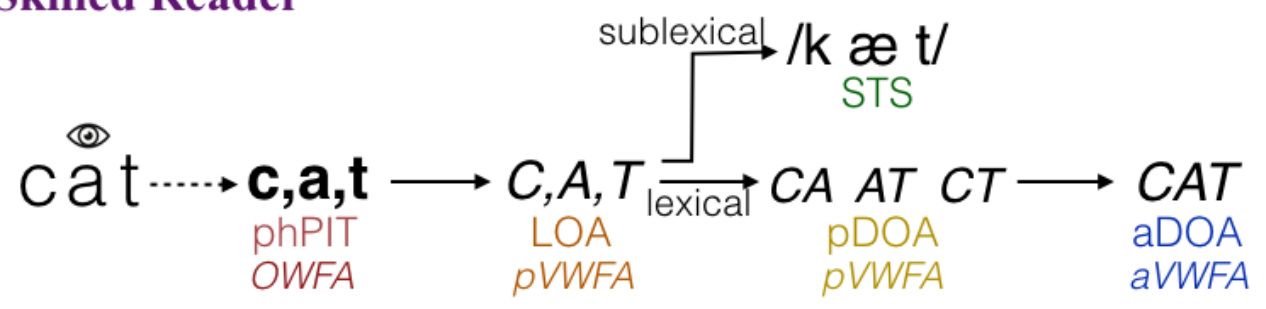

Compensated Dyslexic

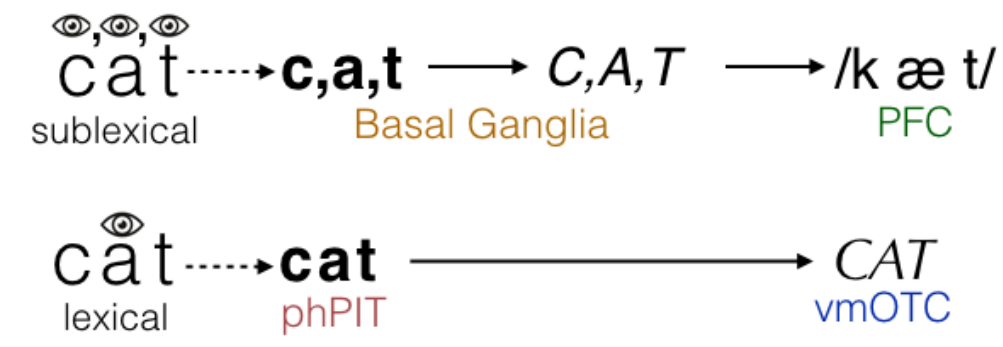

Figure 3: Proposed neural representations for lexical and sublexical processing in skilled and dyslexic readers, illustrated for the stimulus "cat". (Dyslexic processing is addressed in the following section, but displayed here to facilitate comparison with typical readers.) In skilled readers, automatic serial activation of Retinotopic-Letters drives serial Letter activation. On the sublexical pathway, Letters activate Phonemes. On the lexical pathway, Letters activate Open-Bigrams, which activate the corresponding Visual Word Form. In compensated dyslexics, phonological decoding is serial via fixations, while lexical processing is parallel via the vmOTC object-recognition system. $P F C$ Prefrontal Cortex.

This analysis indicates that the VWFA should overlap the DOA within individual subjects. We are currently running an fMRI experiment to test this prediction, and thus far have scanned four adults. The DOA was identifiable in all subjects, via the contrast of dynamic object motion vs human-body motion (Ross, de Gelder, Crabbe, \& Grosbras, 2014). Three subjects' VWFAs fell near the typical location,

195 while the fourth subject's was more anterior than usual. We found VWFA-DOA overlap in the three subjects with typical VWFA locations. Hence, these preliminary data are in line with our claim that the VWFA usually develops as a DOA adaptation for processing sequences of Letters. 


\section{Implications for Developmental Dyslexia}

If VWFA formation does indeed depend on sequential attentional selection of letters, a deficit in such

200 selection would preclude VWFA development and yield dyslexia. However, developmental dyslexia is associated with a persistent strong length effect (especially in languages with a consistent relationship between orthography and phonology) (e.g., Hutzler \& Wimmer, 2004; Martens \& de Jong, 2006; Zoccolotti et al., 2005), indicating slow serial letter selection as in the first orthographic phase. Why doesn't this serial processing induce typical reading acquisition? In the typical first phase, children

205 would narrow attention to the fixated letter. Many children who become dyslexic may be unable to zoom attention to a single letter (Facoetti et al., 2003; Marmurek, 1988; Vidyasagar \& Pammer, 2010). Instead, all the Letters of a short word are simultaneously active in OTC, so the DOA is not recruited to represent Letter sequences. Non-selective Letter activation would also interfere with development of connections between Letters and STS Phonemes, as shown by simulations (Whitney, 2014) that

210 reproduce fMRI patterns of impaired and typical letter-phoneme integration (Blau et al., 2010). However, dyslexics can accurately decode pseudowords, particularly in languages with consistent orthographies. How do they determine the identity of each letter if they cannot attentionally select an individual letter? We suggest that letter selection instead occurs within prefrontal cortex. The Inferior Frontal Junction encodes object features (color, shape, identity) for cognitive control (Baldauf \&

215 Desimone, 2014; Bichot, Heard, DeGennaro, \& Desimone, 2015; Zanto, Rubens, Bollinger, \& Gazzaley, 2010), while Dorsolateral Prefrontal Cortex encodes target location (Rainer, Asaad, \& Miller, 1998; Rowe, Toni, Josephs, Frackowiak, \& Passingham, 2000; Suzuki \& Gottlieb, 2013). The retinotopic inferior Frontal Eye Fields may support a mapping between target location and object identity (Bichot et al., 2015). In children who have difficulty zooming attention, the fixation point

220 may be encoded as the target location in Dorsolateral Prefrontal Cortex, leading to activation of the identity of the fixated letter in the Inferior Frontal Junction. Hence, the fixated letter's identity is determined locally within prefrontal cortex, and multiple Letters remain active in OTC. Figure 4 illustrates typical versus dyslexic phonological decoding in the first orthographic phase.

Over time, such dyslexic phonological decoding would become automated via the procedural system.

225 The Basal Ganglia interact with prefrontal and visual cortex, supporting procedural learning and visuomotor mapping. The tail of the Caudate Nucleus has neurons that encode both object shape and retinal location (Yamamoto, Monosov, Yasuda, \& Hikosaka, 2012; Yeterian \& Pandya, 1995). The Basal Ganglia may learn to determine the identity of the fixated letter and map it to the corresponding 
articulatory representation in premotor cortex. As illustrated in Fig. 4, automatic Letter-Phoneme 230 mapping would be carried out by the procedural system, rather than by OTC as in skilled readers. 

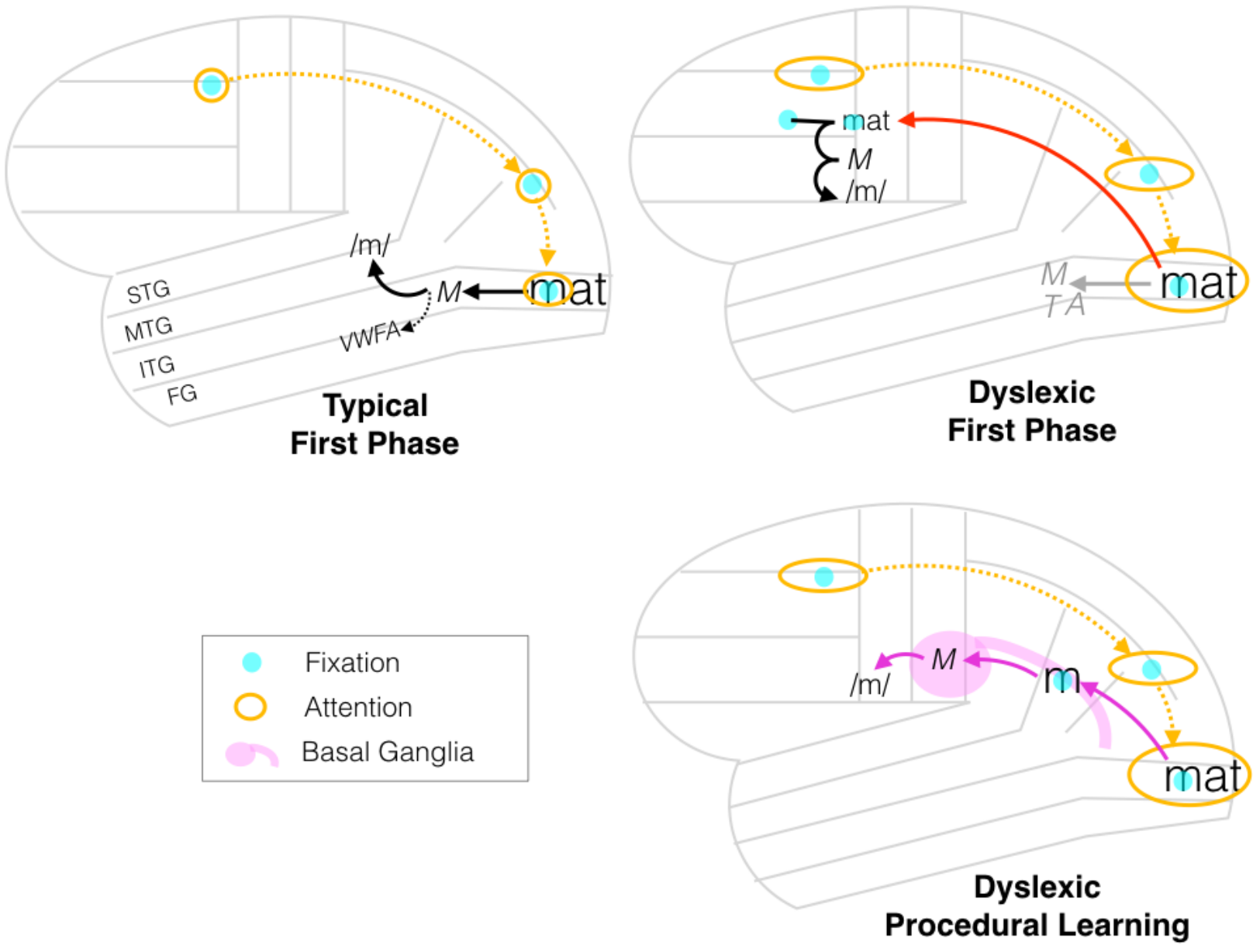

Figure 4: Proposed neural patterns of phonological decoding in typical and atypical readers during early reading acquisition, illustrated for selection of the "m" of the stimulus "mat". Typical First Phase: in children who become skilled readers, attentional selection makes only the Letter $M$ active in vlOTC. Over time, $M$ becomes connected to the Phoneme $/ \mathrm{m} /$ in the STS. Furthermore, the sequence of Letter activations in word decoding drives formation of the VWFA in the DOA. Dyslexic First Phase: in children who become dyslexic, attention cannot be narrowed to a single letter. Rather, the fixated letter is selected and mapped to the corresponding Phoneme within prefrontal cortex. Dyslexic Procedural Learning: over time, this visuomotor mapping (from low-level visual cortex to premotor cortex) is automated via the Basal Ganglia. 
These proposals are consistent with a number of fMRI studies. In impaired readers, Letter processing would primarily take place subcortically, explaining the reduced OTC response to letters (Blau et al., 2010; Boros et al., 2016; Danelli et al., 2017; Peyrin, Démonet, N’Guyen-Morel, Le Bas, \& Valdois,

235 2011). In a task on a non-alphabetic target character at fixation flanked by \#'s, dyslexic subjects showed stronger prefrontal activation than control subjects (Peyrin et al., 2011), in line with our proposal that dyslexics determine the identity of a fixated character within prefrontal cortex. For stimuli in which multiple targets could occur within a string, dyslexics displayed reduced parietal and occipito-parietal activity (Boros et al., 2016; Peyrin et al., 2011), indicating difficulty with top-down attentional 240 selection from prefrontal $\rightarrow$ parietal $\rightarrow$ visual cortex. For pseudoword reading, dyslexics show increased subcortical and premotor activity (Brunswick, McCrory, Price, Frith, \& Frith, 1999; Hancock, Richlan, \& Hoeft, 2017; Richlan et al., 2009, 2010) with visual activity limited to occipital cortex (Danelli et al., 2017), consistent with phonological decoding based on visuomotor mapping through the Basal Ganglia.

245 Because the VWFA does not develop, dyslexics continue to rely on phonological decoding. However, phonological decoding remains slow, as each letter must be fixated (De Luca, Borrelli, Judica, Spinelli, \& Zoccolotti, 2002; Hutzler \& Wimmer, 2004) to be selected. Therefore, many children would eventually give up on phonological decoding, especially in inconsistent orthographies. Instead, they would learn to recognize words in parallel via the vmOTC object-recognition system, becoming

250 "compensated" dyslexics. Accordingly, in a primed lexical-decision task, compensated dyslexics showed morphological, semantic, and orthographic priming in vmOTC (with no priming in vlOTC), while skilled readers showed orthographic priming in vlOTC (with no priming in vmOTC) (Cavalli et al., 2017). However, the vmOTC system is not tuned for representation of linear order. Therefore, compensated dyslexics develop imprecise Visual Word Forms, leading to visual errors in reading and

255 difficulties in spelling. If an unknown word is encountered, letter-by-letter phonological decoding remains necessary. That is, orthographic analyses for lexical and sublexical processing are disjoint in compensated dyslexics, as illustrated in the bottom panel of Fig. 3. In contrast, in skilled readers, pVWFA Letters drive the lexical and sublexical pathways (top panel of Fig. 3).

Hence, if a child remains in the first orthographic phase beyond the first year of reading instruction, an 260 attention-allocation deficit may be the cause. It might be possible to provide remediation by simulating the typical first and second orthographic phases. For example, an app could present words in low contrast, with the contrast of each letter sequentially increased and then decreased. This would 
externally provide the effect of typical top-down attention shifts, while also driving bottom-up attention shifts. Perhaps extensive exposure to such stimuli could jumpstart typical reading development, 265 particularly if commenced early enough.

In conclusion, understanding what goes wrong in developmental dyslexia requires a complete account of normal reading acquisition. The surprising location of the VWFA in a motion-analysis region indicates that serial Letter activation is important in all developmental phases of orthographic processing. A detailed understanding of how the seriality of the first/second phases normally drives 270 acquisition of skilled orthographic analysis is crucial for explaining why dyslexics stagnate at the first phase, and for designing optimal methods of remediation. 
Abdollahi, R. O., Kolster, H., Glasser, M. F., Robinson, E. C., Coalson, T. S., Dierker, D., ... Orban, G. A. (2014). Correspondences between retinotopic areas and myelin maps in human visual cortex. NeuroImage, 99, 509-524. https://doi.org/10.1016/j.neuroimage.2014.06.042

Baldauf, D., \& Desimone, R. (2014). Neural Mechanisms of Object-Based Attention. Science, 344(6182), 424-427. https://doi.org/10.1126/science.1247003

Beauchamp, M. S., Lee, K. E., Haxby, J. V., \& Martin, A. (2002). Parallel Visual Motion Processing Streams for Manipulable Objects and Human Movements. Neuron, 34(1), 149-159. https://doi.org/10.1016/S0896-6273(02)00642-6

Beauchamp, M. S., Lee, K. E., Haxby, J. V., \& Martin, A. (2003). FMRI responses to video and pointlight displays of moving humans and manipulable objects. Journal of Cognitive Neuroscience, 15(7), 991-1001. https://doi.org/10.1162/089892903770007380

Beauchamp, M. S., \& Martin, A. (2007). Grounding Object Concepts in Perception and Action: Evidence from FMRI Studies of Tools. Cortex, 43(3), 461-468. https://doi.org/10.1016/S00109452(08)70470-2

Bichot, N. P., Heard, M. T., DeGennaro, E. M., \& Desimone, R. (2015). A Source for Feature-Based Attention in the Prefrontal Cortex. Neuron, 88(4), 832-844. https://doi.org/10.1016/j.neuron.2015.10.001

Blau, V., Reithler, J., van Atteveldt, N., Seitz, J., Gerretsen, P., Goebel, R., \& Blomert, L. (2010). Deviant processing of letters and speech sounds as proximate cause of reading failure: a functional magnetic resonance imaging study of dyslexic children. Brain: A Journal of Neurology, 133(Pt 3), 868-879. https://doi.org/10.1093/brain/awp308

Blomert, L. (2011). The neural signature of orthographic-phonological binding in successful and failing reading development. NeuroImage, 57(3), 695-703. https://doi.org/10.1016/j.neuroimage.2010.11.003

Boros, M., Anton, J.-L., Pech-Georgel, C., Grainger, J., Szwed, M., \& Ziegler, J. C. (2016). Orthographic processing deficits in developmental dyslexia: Beyond the ventral visual stream. NeuroImage, 128, 316-327. https://doi.org/10.1016/j.neuroimage.2016.01.014 
Bracci, S., Cavina-Pratesi, C., Connolly, J. D., \& Ietswaart, M. (2016). Representational content of occipitotemporal and parietal tool areas. Neuropsychologia, 84, 81-88. https://doi.org/10.1016/j.neuropsychologia.2015.09.001

Bracci, S., Cavina-Pratesi, C., Ietswaart, M., Caramazza, A., \& Peelen, M. V. (2012). Closely overlapping responses to tools and hands in left lateral occipitotemporal cortex. Journal of Neurophysiology, 107(5), 1443-1456. https://doi.org/10.1152/jn.00619.2011

Brunswick, N., McCrory, E., Price, C. J., Frith, C. D., \& Frith, U. (1999). Explicit and implicit processing of words and pseudowords by adult developmental dyslexicsA search for Wernicke’s Wortschatz? Brain, 122(10), 1901-1917. https://doi.org/10.1093/brain/122.10.1901

Cavalli, E., Colé, P., Pattamadilok, C., Badier, J.-M., Zielinski, C., Chanoine, V., \& Ziegler, J. C. (2017). Spatiotemporal reorganization of the reading network in adult dyslexia. Cortex, 92, 204-221. https://doi.org/10.1016/j.cortex.2017.04.012

Cohen, L., Jobert, A., Le Bihan, D., \& Dehaene, S. (2004). Distinct unimodal and multimodal regions for word processing in the left temporal cortex. NeuroImage, 23(4), 1256-1270. https://doi.org/ 10.1016/j.neuroimage.2004.07.052

Costantini, M., Galati, G., Ferretti, A., Caulo, M., Tartaro, A., Romani, G. L., \& Aglioti, S. M. (2005). Neural Systems Underlying Observation of Humanly Impossible Movements: An fMRI Study. Cerebral Cortex, 15(11), 1761-1767. https://doi.org/10.1093/cercor/bhi053

Danelli, L., Berlingeri, M., Bottini, G., Borghese, N. A., Lucchese, M., Sberna, M., ... Paulesu, E. (2017). How many deficits in the same dyslexic brains? A behavioural and fMRI assessment of comorbidity in adult dyslexics. Cortex, 97, 125-142. https://doi.org/10.1016/j.cortex.2017.08.038

Danelli, L., Berlingeri, M., Bottini, G., Ferri, F., Vacchi, L., Sberna, M., \& Paulesu, E. (2013). Neural intersections of the phonological, visual magnocellular and motor/cerebellar systems in normal readers: Implications for imaging studies on dyslexia. Human Brain Mapping, 34(10), 26692687. https://doi.org/10.1002/hbm.22098 
De Luca, M., Borrelli, M., Judica, A., Spinelli, D., \& Zoccolotti, P. (2002). Reading Words and Pseudowords: An Eye Movement Study of Developmental Dyslexia. Brain and Language, 80(3), 617-626. https://doi.org/10.1006/brln.2001.2637

De Luca, M., Zeri, F., Spinelli, D., \& Zoccolotti, P. (2010). The acquisition of reading fluency in an orthographically transparent language (Italian): an eye movement longitudinal study. Medical Science Monitor: International Medical Journal of Experimental and Clinical Research, 16(3), SC1-7.

Dehaene, S., \& Cohen, L. (2011). The unique role of the visual word form area in reading. Trends in Cognitive Sciences, 15(6), 254-262. https://doi.org/10.1016/j.tics.2011.04.003

Dehaene, S., Jobert, A., Naccache, L., Ciuciu, P., Poline, J.-B., Le Bihan, D., \& Cohen, L. (2004). Letter binding and invariant recognition of masked words: behavioral and neuroimaging evidence. Psychological Science: A Journal of the American Psychological Society / APS, 15(5), 307-313. https://doi.org/10.1111/j.0956-7976.2004.00674.x

Dehaene-Lambertz, G., Monzalvo, K., \& Dehaene, S. (2018). The emergence of the visual word form: Longitudinal evolution of category-specific ventral visual areas during reading acquisition. PLOS Biology, 16(3), e2004103. https://doi.org/10.1371/journal.pbio.2004103

Downing, P. E., Jiang, Y., Shuman, M., \& Kanwisher, N. (2001). A Cortical Area Selective for Visual Processing of the Human Body. Science, 293(5539), 2470-2473. https://doi.org/10.1126/science.1063414

Facoetti, A., Lorusso, M. L., Paganoni, P., Cattaneo, C., Galli, R., \& Mascetti, G. G. (2003). The time course of attentional focusing in dyslexic and normally reading children. Brain and Cognition, 53(2), 181-184.

Gagl, B., Hawelka, S., \& Wimmer, H. (2015). On Sources of the Word Length Effect in Young Readers. Scientific Studies of Reading, 19(4), 289-306. https://doi.org/10.1080/10888438.2015.1026969 
Glezer, L. S., Jiang, X., \& Riesenhuber, M. (2009). Evidence for highly selective neuronal tuning to whole words in the “visual word form area.” Neuron, 62(2), 199-204. https://doi.org/10.1016/j.neuron.2009.03.017

Glezer, L. S., Kim, J., Rule, J., Jiang, X., \& Riesenhuber, M. (2015). Adding Words to the Brain’s Visual Dictionary: Novel Word Learning Selectively Sharpens Orthographic Representations in the VWFA. Journal of Neuroscience, 35(12), 4965-4972. https://doi.org/10.1523/JNEUROSCI.4031-14.2015

Grill-Spector, K. (2003). The neural basis of object perception. Current Opinion in Neurobiology, 13(2), 159-166. https://doi.org/10.1016/S0959-4388(03)00040-0

Hancock, R., Richlan, F., \& Hoeft, F. (2017). Possible roles for fronto-striatal circuits in reading disorder. Neuroscience \& Biobehavioral Reviews, 72, 243-260.

https://doi.org/10.1016/j.neubiorev.2016.10.025

Hasson, U., Harel, M., Levy, I., \& Malach, R. (2003). Large-Scale Mirror-Symmetry Organization of Human Occipito-Temporal Object Areas. Neuron, 37(6), 1027-1041. https://doi.org/10.1016/S0896-6273(03)00144-2

Hutzler, F., \& Wimmer, H. (2004). Eye movements of dyslexic children when reading in a regular orthography. Brain and Language, 89(1), 235-242. https://doi.org/10.1016/S0093934X(03)00401-2

Ishai, A., Ungerleider, L. G., Martin, A., Schouten, J. L., \& Haxby, J. V. (1999). Distributed representation of objects in the human ventral visual pathway. Proceedings of the National Academy of Sciences, 96(16), 9379-9384. https://doi.org/10.1073/pnas.96.16.9379

James, K. H., James, T. W., Jobard, G., Wong, A. C. N., \& Gauthier, I. (2005). Letter processing in the visual system: different activation patterns for single letters and strings. Cognitive, Affective \& Behavioral Neuroscience, 5(4), 452-466.

Jastorff, J., \& Orban, G. A. (2009). Human Functional Magnetic Resonance Imaging Reveals Separation and Integration of Shape and Motion Cues in Biological Motion Processing. Journal of Neuroscience, 29(22), 7315-7329. https://doi.org/10.1523/JNEUROSCI.4870-08.2009 
Kanwisher, N., McDermott, J., \& Chun, M. M. (1997). The Fusiform Face Area: A Module in Human Extrastriate Cortex Specialized for Face Perception. The Journal of Neuroscience, 17(11), 4302-4311.

Kolster, H., Peeters, R., \& Orban, G. A. (2010). The retinotopic organization of the human middle temporal area MT/V5 and its cortical neighbors. The Journal of Neuroscience: The Official Journal of the Society for Neuroscience, 30(29), 9801-9820.

https://doi.org/10.1523/JNEUROSCI.2069-10.2010

Konkle, T., \& Caramazza, A. (2013). Tripartite Organization of the Ventral Stream by Animacy and Object Size. Journal of Neuroscience, 33(25), 10235-10242. https://doi.org/10.1523/JNEUROSCI.0983-13.2013

Lafer-Sousa, R., Conway, B. R., \& Kanwisher, N. G. (2016). Color-Biased Regions of the Ventral Visual Pathway Lie between Face- and Place-Selective Regions in Humans, as in Macaques. Journal of Neuroscience, 36(5), 1682-1697. https://doi.org/10.1523/JNEUROSCI.316415.2016

Lerma-Usabiaga, G., Carreiras, M., \& Paz-Alonso, P. M. (2018). Converging evidence for functional and structural segregation within the left ventral occipitotemporal cortex in reading. Proceedings of the National Academy of Sciences of the United States of America, 115(42), E9981-E9990. https://doi.org/10.1073/pnas.1803003115

Liu, J., Harris, A., \& Kanwisher, N. (2010). Perception of face parts and face configurations: an FMRI study. Journal of Cognitive Neuroscience, 22(1), 203-211. https://doi.org/10.1162/jocn.2009.21203

Marinus, E., \& de Jong, P. F. (2010). Variability in the word-reading performance of dyslexic readers: effects of letter length, phoneme length and digraph presence. Cortex; a Journal Devoted to the Study of the Nervous System and Behavior, 46(10), 1259-1271. https://doi.org/10.1016/j.cortex.2010.06.005

Marmurek, H. H. C. (1988). Reading Ability and Attention to Words and Letters in Words. Journal of Reading Behavior, 20(2), 119-129. https://doi.org/10.1080/10862968809547630 
Martens, V. E. G., \& de Jong, P. F. (2006). The effect of word length on lexical decision in dyslexic and normal reading children. Brain and Language, 98(2), 140-149. https://doi.org/10.1016/j.bandl.2006.04.003

Monzalvo, K., Fluss, J., Billard, C., Dehaene, S., \& Dehaene-Lambertz, G. (2012). Cortical networks for vision and language in dyslexic and normal children of variable socio-economic status. NeuroImage, 61(1), 258-274. https://doi.org/10.1016/j.neuroimage.2012.02.035

Paulesu, E., Danelli, L., \& Berlingeri, M. (2014). Reading the dyslexic brain: multiple dysfunctional routes revealed by a new meta-analysis of PET and fMRI activation studies. Frontiers in Human Neuroscience, 8. https://doi.org/10.3389/fnhum.2014.00830

Peelen, M. V., \& Downing, P. E. (2005). Selectivity for the Human Body in the Fusiform Gyrus. Journal of Neurophysiology, 93(1), 603-608. https://doi.org/10.1152/jn.00513.2004

Peyrin, C., Démonet, J. F., N’Guyen-Morel, M. A., Le Bas, J. F., \& Valdois, S. (2011). Superior parietal lobule dysfunction in a homogeneous group of dyslexic children with a visual attention span disorder. Brain and Language, 118(3), 128-138. https://doi.org/10.1016/j.bandl.2010.06.005

Polosecki, P., Moeller, S., Schweers, N., Romanski, L. M., Tsao, D. Y., \& Freiwald, W. A. (2013). Faces in Motion: Selectivity of Macaque and Human Face Processing Areas for Dynamic Stimuli. Journal of Neuroscience, 33(29), 11768-11773. https://doi.org/10.1523/JNEUROSCI.540211.2013

Pyles, J. A., Garcia, J. O., Hoffman, D. D., \& Grossman, E. D. (2007). Visual perception and neural correlates of novel 'biological motion.' Vision Research, 47(21), 2786-2797. https://doi.org/10.1016/j.visres.2007.07.017

Rainer, G., Asaad, W. F., \& Miller, E. K. (1998). Selective representation of relevant information by neurons in the primate prefrontal cortex. Nature, 393(6685), 577-579. https://doi.org/10.1038/31235

Richlan, F., Kronbichler, M., \& Wimmer, H. (2009). Functional abnormalities in the dyslexic brain: a quantitative meta-analysis of neuroimaging studies. Human Brain Mapping, 30(10), 32993308. https://doi.org/10.1002/hbm.20752 
Richlan, F., Sturm, D., Schurz, M., Kronbichler, M., Ladurner, G., \& Wimmer, H. (2010). A common left occipito-temporal dysfunction in developmental dyslexia and acquired letter-by-letter reading? PloS One, 5(8), e12073. https://doi.org/10.1371/journal.pone.0012073

Ross, P. D., de Gelder, B., Crabbe, F., \& Grosbras, M.-H. (2014). Body-selective areas in the visual cortex are less active in children than in adults. Frontiers in Human Neuroscience, 8. https://doi.org/10.3389/fnhum.2014.00941

Rowe, J. B., Toni, I., Josephs, O., Frackowiak, R. S. J., \& Passingham, R. E. (2000). The Prefrontal Cortex: Response Selection or Maintenance Within Working Memory? Science, 288(5471), 1656-1660. https://doi.org/10.1126/science.288.5471.1656

Silson, E. H., McKeefry, D. J., Rodgers, J., Gouws, A. D., Hymers, M., \& Morland, A. B. (2013). Specialized and independent processing of orientation and shape in visual field maps LO1 and LO2. Nature Neuroscience, 16(3), 267-269. https://doi.org/10.1038/nn.3327

Spiridon, M., Fischl, B., \& Kanwisher, N. (2006). Location and Spatial Profile of Category-Specific Regions in Human Extrastriate Cortex. Human Brain Mapping, 27(1), 77-89. https://doi.org/10.1002/hbm.20169

Stevens, W. D., Tessler, M. H., Peng, C. S., \& Martin, A. (2015). Functional connectivity constrains the category-related organization of human ventral occipitotemporal cortex. Human Brain Mapping, 36(6), 2187-2206. https://doi.org/10.1002/hbm.22764

Strother, L., Coros, A. M., \& Vilis, T. (2016). Visual Cortical Representation of Whole Words and Hemifield-split Word Parts. Journal of Cognitive Neuroscience, 28(2), 252-260. https://doi.org/ 10.1162/jocn_a_00900

Strother, L., Zhou, Z., Coros, A. K., \& Vilis, T. (2017). An fMRI study of visual hemifield integration and cerebral lateralization. Neuropsychologia, 100, 35-43. https://doi.org/10.1016/j.neuropsychologia.2017.04.003

Suzuki, M., \& Gottlieb, J. (2013). Distinct neural mechanisms of distractor suppression in the frontal and parietal lobe. Nature Neuroscience, 16(1), 98-104. https://doi.org/10.1038/nn.3282 
Thesen, T., McDonald, C. R., Carlson, C., Doyle, W., Cash, S., Sherfey, J., ... Halgren, E. (2012).

Sequential then interactive processing of letters and words in the left fusiform gyrus. Nature Communications, 3, 1284. https://doi.org/10.1038/ncomms2220

Vangeneugden, J., Peelen, M. V., Tadin, D., \& Battelli, L. (2014). Distinct neural mechanisms for body form and body motion discriminations. The Journal of Neuroscience: The Official Journal of the Society for Neuroscience, 34(2), 574-585. https://doi.org/10.1523/JNEUROSCI.403213.2014

Vidyasagar, T. R., \& Pammer, K. (2010). Dyslexia: a deficit in visuo-spatial attention, not in phonological processing. Trends in Cognitive Sciences, 14(2), 57-63. https://doi.org/10.1016/j.tics.2009.12.003

Vinckier, F., Dehaene, S., Jobert, A., Dubus, J. P., Sigman, M., \& Cohen, L. (2007). Hierarchical coding of letter strings in the ventral stream: dissecting the inner organization of the visual word-form system. Neuron, 55(1), 143-156. https://doi.org/10.1016/j.neuron.2007.05.031

Weiner, K. S., \& Grill-Spector, K. (2013). Neural representations of faces and limbs neighbor in human high-level visual cortex: evidence for a new organization principle. Psychological Research, 77(1), 74-97. https://doi.org/10.1007/s00426-011-0392-x

Whitney, C. (2001). How the brain encodes the order of letters in a printed word: the SERIOL model and selective literature review. Psychonomic Bulletin \& Review, 8(2), 221-243.

Whitney, C. (2014). Simulating a deficit in grapheme-phoneme integration. Retrieved from http://www.researchgate.net/publication/259931382_Simulating_a_Deficit_in_GraphemePhoneme_Integration.pdf

Whitney, C. (2018). When serial letter processing implies a facilitative length effect. Language, Cognition and Neuroscience, 33(5), 659-664. https://doi.org/10.1080/23273798.2017.1404115 Whitney, C., \& Marton, Y. (2013). The SERIOL2 model of orthographic processing. Retrieved from http://files.eric.ed.gov/fulltext/ED543279.pdf 
Whitney, C., Ross, P., Zhou, Z., \& Strother, L. (2019). A novel hypothesis for the original functionality of the Visual Word Form Area: Processing shape sequences. https://doi.org/10.31234/osf.io/g3n2m

Wiggett, A. J., Pritchard, I. C., \& Downing, P. E. (2009). Animate and inanimate objects in human visual cortex: Evidence for task-independent category effects. Neuropsychologia, 47(14), 31113117. https://doi.org/10.1016/j.neuropsychologia.2009.07.008

Yamamoto, S., Monosov, I. E., Yasuda, M., \& Hikosaka, O. (2012). What and Where Information in the Caudate Tail Guides Saccades to Visual Objects. Journal of Neuroscience, 32(32), 1100511016. https://doi.org/10.1523/JNEUROSCI.0828-12.2012

Yeterian, E. H., \& Pandya, D. N. (1995). Corticostriatal connections of extrastriate visual areas in rhesus monkeys. The Journal of Comparative Neurology, 352(3), 436-457. https://doi.org/10.1002/cne.903520309

Zanto, T. P., Rubens, M. T., Bollinger, J., \& Gazzaley, A. (2010). Top-down modulation of visual feature processing: The role of the inferior frontal junction. NeuroImage, 53(2), 736-745. https://doi.org/10.1016/j.neuroimage.2010.06.012

Zoccolotti, P., De Luca, M., Di Pace, E., Gasperini, F., Judica, A., \& Spinelli, D. (2005). Word length effect in early reading and in developmental dyslexia. Brain and Language, 93(3), 369-373. https://doi.org/10.1016/j.bandl.2004.10.010 